\title{
Draft Genome Sequence of Enterobacter hormaecheiST93, a Clinical Tigecycline Resistant Strain Harbouring blaNDM-1
}

\section{Lia $X^{1}$, Sunb $L^{2}$, Zhua $Y^{1}$ and Wang $D^{3 *}$}

${ }^{1}$ Centre of Laboratory Medicine, Zhejiang Provincial People's Hospital, People's Hospital of Hangzhou Medical College, Hangzhou, Zhejiang China

${ }^{2}$ Department of Clinical Laboratory, Hangzhou Hospital of Zhejiang Provincial Corps, Chinese People's Armed Police Forces, Hangzhou China

${ }^{3}$ Blood Center of Zhejiang Province, Hangzhou, Zhejiang, China

*Corresponding author: Wang D, Blood Center of Zhejiang Province, 789 Jianye Road, Hangzhou, Zhejiang 310052, China, Tel: +86-571-8589-3264; E-mail: jywangdairong@163.com

Received date: March 19, 2018; Accepted date: April 11, 2018; Published date: April 16, 2018

Copyright: $@ 2018$ Lia X, et al. This is an open-access article distributed under the terms of the Creative Commons Attribution License, which permits unrestricted use, distribution, and reproduction in any medium, provided the original author and source are credited.

\begin{abstract}
Objective: The emergence of Carbapenem-resistant $E$. cloacae complex has been a serious challenge to manage in the clinic because of multi-drug resistance. Tigecycline is regarded as one of the last-resort for Carbapenem-resistant E. cloacae complex infections. Here, we report the draft genome sequence of a tigecycline resistant NDM-1-producing $E$. hormaechei strain ETR1 that was isolated from a male in China.

Methods: Whole-genomic DNA of E. hormaechei strain ETR1 was extracted and was sequenced using an Illumina-Hiseq ${ }^{\mathrm{TM}} \mathrm{X}$ Ten platform. The generated sequence reads were assembled using CLC Genomics Workbench. The draft genome was annotated using Rapid Annotation using Subsystem Technology. Bioinformatics analysis was further performed.
\end{abstract}

Results: The 5,141,975 bp genome contains various antimicrobial resistance genes conferring resistance to beta-lactam, fosfomycin, fluoroquinolone and tetracycline. Notably, the strain was also resistant to carbapenem and tigecycline. In addition, virulence factor encoding genes were also identified.

Conclusion: The genome sequence will provide valuable information to understand antibiotic resistance mechanisms and pathogenic mechanisms in this strain. Close surveillance is urgently needed to monitor the spread of tigecycline resistant NDM-1-producing isolates.

Keywords: Enterobacter cloacae complex; NDM-1; Whole-genome sequencing

\section{Introduction}

Enterobacter cloacae complex is an opportunistic pathogen that can cause a variety of infections, especially in immune-compromised individuals. Moreover, it is able to carry a number of antibiotic resistance genes, which make it a difficult-to-treat pathogen [1]. There is a growing recognition that $E$. cloacae complex is now the third major Enterobacteriaceae species associated with healthcare-associated infections after Escherichia coli and Klebsiella pneumoniae [2]. It is worrying that (New Delhi metallo- $\beta$-lactamase) NDM-producing $E$. cloacae complex has reported during recent years, limiting the therapeutic options [1]. Tigecycline is regarded as one of the last-resort for CRE (carbapenem-resistant Enterobacteriaceae, CRE) infections. However, the emergence of tigecycline resistant CRE strains has been a serious challenge to public health.

\section{Methods}

In addition, few studies reported tigecycline resistant $E$. hormaechei strains. In the present study, the whole genome sequence of a clinical tigecycline resistant $E$. hormaechei sequence type 93 strain producing
NDM-1 carbapenemases was identified to understand antibiotic resistance and pathogenic mechanisms.

Strain E. hormaechei ETR1 was recovered from an ascites sample of a male patient who was admitted to the hospital for gall-stone in Hangzhou, Zhejiang Province, China, in 2016. The strain was grown overnight at $37^{\circ} \mathrm{C}$ in $\mathrm{MH}$ agar (Oxoid, United Kingdom). Strain $E$. hormaechei ETR1 was preliminarily identified by the VITEK 2 system (Sysmex-bioMérieux, Marcy l'Etoile, France) and further confirmed by $16 S$ rRNA gene sequencing analysis. Antimicrobial susceptibility testing was performed by agar dilution or E-test method. All susceptibility tests were repeated at least three times and MICs were interpreted according to the CLSI guidelines, except tigecycline and polymyxin B, for which European Committee on Antimicrobial Susceptibility Testing breakpoints were used [3]. Whole-genomic DNA of this strain was extracted using a QIAamp DNA MiniKit (Qiagen, Valencia, CA, USA) following the manufacturer's recommendations. The genome was sequenced on an Illumina-Hiseq ${ }^{\mathrm{TM}} \mathrm{X}$ Ten (Illumina Inc, San Diego, U.S.A) using a paired-end $2 \times 100$ bp protocol. Sequence reads were assembled using CLC Genomics Workbench v.10.0 software (QIAGEN, Hilden, Germany). The contigs were initially annotated using Rapid Annotation using Subsystem Technology (RAST) and were then manually checked. Resistance and virulence genes, the sequence type (ST) and plasmid Inc type of the strain were analysed using ResFinder 3.0, Virulence Finder 1.5, MLST 1.8 server and PlasmidFinder 1.3, which are available at the Center for Genomic 
Citation: Lia X, Sunb L, Zhua Y, Wang D (2018) Draft Genome Sequence of Enterobacter hormaechei ST93, a Clinical Tigecycline Resistant Strain Harbouring blaNDM-1. J Antimicrob Agents 4: 167. doi:10.4172/2472-1212.1000167

Page 2 of 2

Epidemiology [4]. In addition, the ISfinder, CRISPRFinder and PHAST databases were used to characterize insertion elements (ISs), clustered regularly interspaced short palindromic repeats (CRISPRs) and prophage sequences, respectively, of strain E. hormaechei ETR1.

\section{Discussion}

Strain ETR1 was resistant to the majority of the antimicrobial agents tested, including ampicillin/sulbactam, aztreonam, ceftazidime, cefepime, ceftriaxone, ertapenem, cefotetan, imipenem, piperacillin/ tazobactam, ciprofloxacin, levofloxacin, minocycline, tetracycline and tigecycline, with exception of polymyxin E and classified MDR bacteria according to the recently proposed international classification scheme [5].

The draft genome consists of 5,141,975 bp with an N50 value of 155,713 bp with a $54.7 \%$ GC content. Using RAST analysis, 4671 ORFs and 80 RNAs gene were predicted, of which 3836 (82.1\%) could be functionally annotated (Figure 1). The distribution of subsystems in this strain was described by RAST server based annotation of the whole genome. Proteins responsible for carbohydrates (651 ORFs), derivatives and amino acids (466 ORFs), and cofactors, vitamins, prosthetic groups and pigments (261 ORFs) were ample among the subsystem categories.
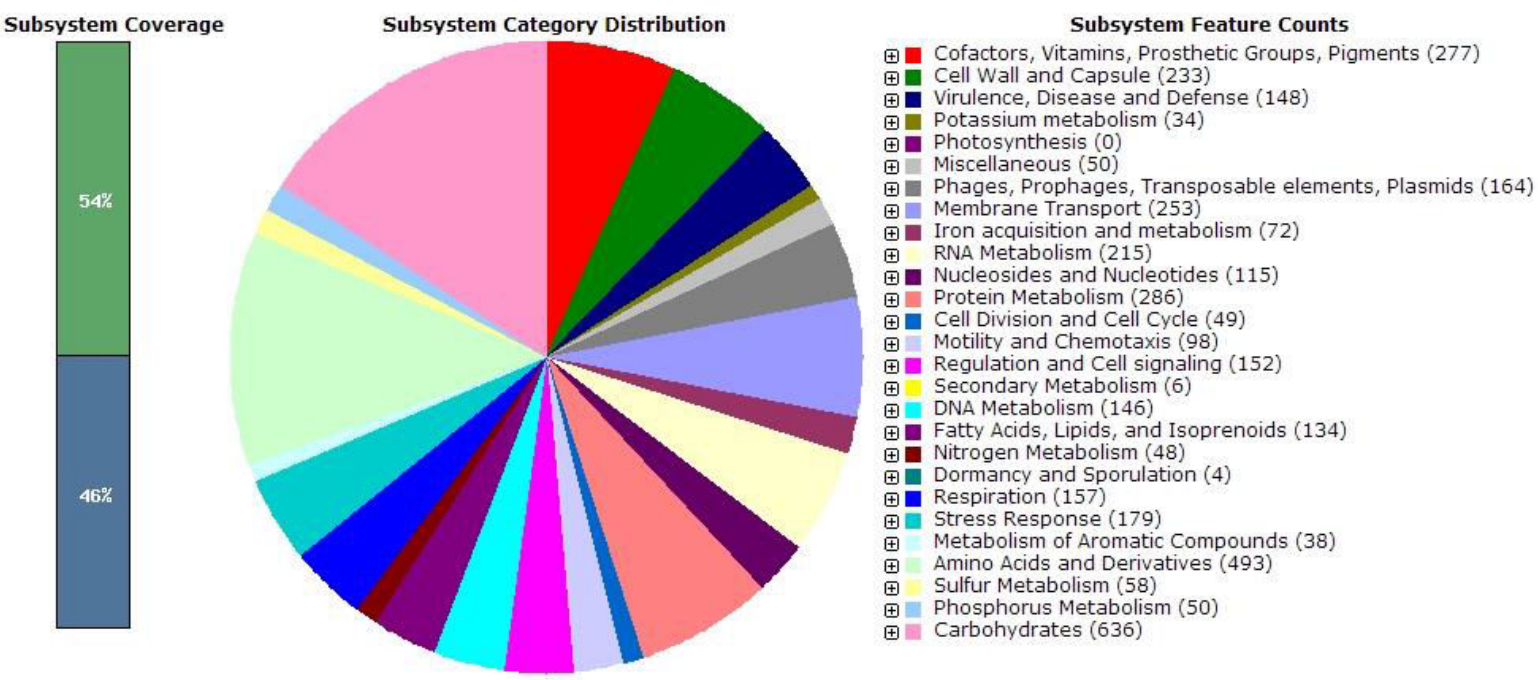

Figure 1: Subsystem category distribution of E. cloacae HBY based on SEED databases.

Resistance genes to beta-lactam (blaACT-7, blaNDM-1 and blaSHV-12), fosfomycin (fos $A$ ), fluoroquinoloneoqx $B$ and oqxAand tetracycline (tet34) were identified. Furthermore, southern blot showed that the blaNDM-1 gene was located in the IncX3 plasmid (ca. 50k). Tigecycline resistance in strain E. hormaechei ETR1 may be mediated by efflux pump system because RND and MFS family multidrug transport proteins were identified in this genome. MLST analysis showed that the strain belonged to ST93. Plasmid Inc types of IncQ1 and IncX3 were also identified. In addition, the genome contains several IS elements, the majority belonging to the IS3, IS21, IS66 and IS630 families. Similarly, there are five complete and three incomplete prophage sequences, and two CRISPR sequences that can be predicted in the genome. In addition, the type 1 fimbriae genes fimABCDEFGHI, encoding virulence determinants, were identified by the RAST annotation. Furthermore, type IV fimbrial genes pilABC were also identified. Other virulence genes for hyperadherence (yid $\mathrm{E}$ ), virulence protein $(M s g A)$ and several putative virulence factors were also identified in this genome. The identified virulence determinants might have contributed to the infection or colonization of this strain.

\section{Conclusion}

In conclusion, whole genome sequening and the results of the bioinformatics analysis of this strain provide a basis for understanding the resistance and pathogenic mechanisms. Furthermore, this information will be useful for raising awareness of the emergence of a tigecycline resistant $E$. hormaechei strain from China producing blaNDM-1.

\section{References}

1. Sanders WE Jr, Sanders CC (1997) Enterobacter spp.: Pathogens poised to flourish at the turn of the century. Clin Microbiol Rev 10: 220-241.

2. Moradigaravand D, Reuter S, Martin V, Peacock SJ, Parkhill J (2016) The dissemination of multidrug-resistant Enterobacter cloacae throughout the UK and Ireland. Nat Microbiol 1: 16173.

3. CLSI performance standards for antimicrobial susceptibility testing; 27th edition (2017). CLSI supplement M100. Wayne, PA: Clinical and Laboratory Standards Institute.

4. Zankari E, Hasman H, Cosentino S, Vestergaard M, Rasmussen S, et al. (2012) Identification of acquired antimicrobial resistance genes. J Antimicrob Chemother 67: 2640-2644.

5. Magiorakos AP, Srinivasan A, Carey RB, Carmeli Y, Falagas ME, et al. (2012) Multidrug-resistant, extensively drug-resistant and pandrugresistant bacteria: An international expert proposal for interim standard definitions for acquired resistance. Clin microbiol infect 18: 268-281. 